\title{
A Novel Optimal Distributed Generation Planning in Distribution Network using Cuckoo Optimization Algorithm
}

\author{
Ali Aranizadeh, Iman Niazazari, and Mirpouya Mirmozaffari*
}

\begin{abstract}
The optimal sizing and placement of distributed generators have recently drawn a considerable attention to itself. This paper proposes an evolutionary cuckoo optimization algorithm (COA) for optimal placement of distributed generation (DG) in a distribution system. The optimal DG placement problem is formulated as a cost function of network losses, voltage profile, and DG expenses. The proposed method is validated on a 13-bus distribution system. The results show that any variation in the parameter's weight in the objective function leads to a significant change in the prediction of the DG's location and capacity.
\end{abstract}

Index Terms - Cuckoo optimization algorithm, distributed generation, fuel cost, network loss, voltage profile

\section{INTRODUCTION}

Distributed generations (DGs) have assisted the power grids via compensating the shortage of power from the grid's primary generation resources by injecting the active and reactive powers to the network. They are beneficial in terms of increasing the power system reliability, enhancing power quality, and reducing the peak power requirements. [1]. These resources usually are installed in the low voltage part of the grid and help to improve voltage profile and reduce network losses.

Many researchers have studied several topics using various optimization techniques to examine the parameters of the objective function to improve the network. In [2], the authors used genetic algorithms for renewable energy sources benefit to improve the voltage profile and increase the load ability rate. Authors in [3] introduced the novel concept of the energy price tag, and the results show an efficient reduction of electricity bill and charging costs of energy storage systems through SFS optimization. In [4], the authors optimized the wind turbine length using the genetic algorithm (GA) to reduce loss in the network. In [5], the authors improved the voltage profile and reduced power losses in the network by load flow analysis and specifying the voltage collapse of sensitive buses. A fuzzy logic based method for reducing losses using a conduit hydropower generator was proposed in [6]. This method has been tested and verified on an actual test system. In [7], the authors improved the voltage profile and load ability factor of the network by optimizing the charging pattern of plug-in hybrid electric vehicles (PHEV) in charging stations. Reference [8] used optimization reactive power flow (ORPF) in the network and the immune algorithm (IA) to improve voltage stability.

Reference [9] used different placement and sizing of distributed generation sources to minimize power system losses due to the difference between load models in the distribution system. References [10]-[13] analyzed the reactive power market by using simultaneous DG and capacitor placement and sizing in a radial distribution system. Several studies have investigated the optimal placement and sizing of parallel flexible AC transmission system (FACTS) elements in the network, and focused on improving the load factor and voltage profile [14]-[16]. In [17], a method was introduced for improving the efficiency of a microgrid with high integration of DGs which can work in both grid connected and islanded mode of operation. The method is capable of detecting and classifying faults quickly so that the system can be recovered in a very short time. Reference [18] used the voltage constraint, feeders' capacity and DG sources level to improve voltage stability in the distribution system based on placement and sizing. Reference [19] focuses on optimization of microgrid frequency profile in presence of uncertainties of DGs, according to environmental and economic objectives.

Reference [20] presents a multi-objective firework optimization algorithm for economic dispatch in microgrids and the results are compared with non-dominated sorting genetic algorithm II (NSGA-II). Reference [21] investigated the optimal placement and sizing of DG by comparing the particle swarm optimization (PSO) and the GA methods with aim at reducing network losses and improving the voltage profile. By increasing injected power of DGs to the grid, reference [22] focused on network losses, system load ability factor, and buses voltage constraints. Reference [23] proposed a new index to determine the optimal size and location of DG units, in order to minimize active power losses and enhance voltage stability margin with considering load variations. Other optimization algorithms can be addressed through decision tree in data mining in reference [24]. Fuzzy expert system plays an important role in optimization in reference [25]. In order to find best performance Decision Making Units (DMUs), Data Envelopment Analysis (DEA) in operation research play an important role in optimization too [26]. This paper presents a novel optimal DG placement approach using cuckoo optimization algorithm (COA).

The rest of this paper is organized as follows: Section 2 provides a mathematical model of the problem. Section 3 
presents the methodology followed by the simulation results in section 4. Finally, the conclusion is presented in section 5 .

\section{PROBLEM FORMULATION}

In this paper, the location and capacity of DGs are used to minimize the objective function. The objective function used in this paper has three parameters: voltage profile, network losses, and DG costs. In this paper, we used the (COA) to show the effect of any change in the weight of each parameter on the placement and DG capacity. This relationship is expressed in (1). The most important purpose of this paper is to show how much impact each of these parameters has on determining placement and DG capacity.

$$
O F=K_{1} \times P_{\text {Loss }}+K_{2} \times V S I+K_{3} \times \operatorname{Cost}_{D G}
$$

where $\mathrm{K}_{1}$ is the weight factor of the loss reduction parameter, $K_{2}$ is the weight factor of the voltage profile parameter and $\mathrm{K}_{3}$ is the weight factor of the fuel cost parameter. In addition, we have the summation of these three weight factors are unity. $P_{\text {Loss }}$ shows the amount of losses. This relationship is expressed in (2). When active power is injected to one bus by a DG, power flow in another line decreases. As a result, the total power loss is decreased in the system.

$$
P_{\text {Loss }}=\sum_{i=1}^{n} R_{i j}\left|I_{i j}\right|^{2}
$$

Moreover, the normal voltage range in each bus is intended to be between 0.95 to $1.05 \mathrm{pu}$. If the voltage stays in this range, the second term in (1) decreases. On the other hand, the voltage increases if the voltage value is outside this range. This relationship is expressed in (3).

$$
V S I=\left|\left(\frac{V-V_{\text {nom }}}{0.05}\right)^{2}\right|
$$

Finally, the last term is related to the DG fuel costs.

\section{METHODOLGY}

The cuckoo optimization algorithm (COA) is inspired by the life of the cuckoo and used a powerful tool in optimization problems. Fig. 1 shows the proposed flowchart of COA. In this algorithm, the process starts with an initial population of cuckoos. The cuckoo populations place some eggs in some host bird's nests. Some of these eggs, which are more similar to the host bird's eggs, have the opportunity to hatch and become a mature cuckoo. Obviously, other eggs are recognized and discarded from the nest. The habitat in which more eggs survive will be the term that COA is going to optimize. Cuckoos look for the best nest to increase their eggs survival. After the chicks are hatched and become mature, they form new societies of cuckoos in that area. Now, each society has its specific habitat for the living. The best habitat for all societies will be the next goal for the cuckoos in other societies. Hence, all societies fly to the best habit. Each society will be living in a region near the best habitat. Considering the number of eggs that each cuckoo will lay and DOI: http://dx.doi.org/10.24018/ejece.2019.3.3.82 the distance of cuckoos from the current optimal region for residence, a number of possible egg laying radiuses are calculated and formed. Then, cuckoos start to randomly lay eggs in the nests inside these laying radiuses. This process continues until the cuckoo reaches the best place to lay its eggs, i.e., the region with the highest profit. This optimum location is where the highest number of cuckoos will congregate [27].

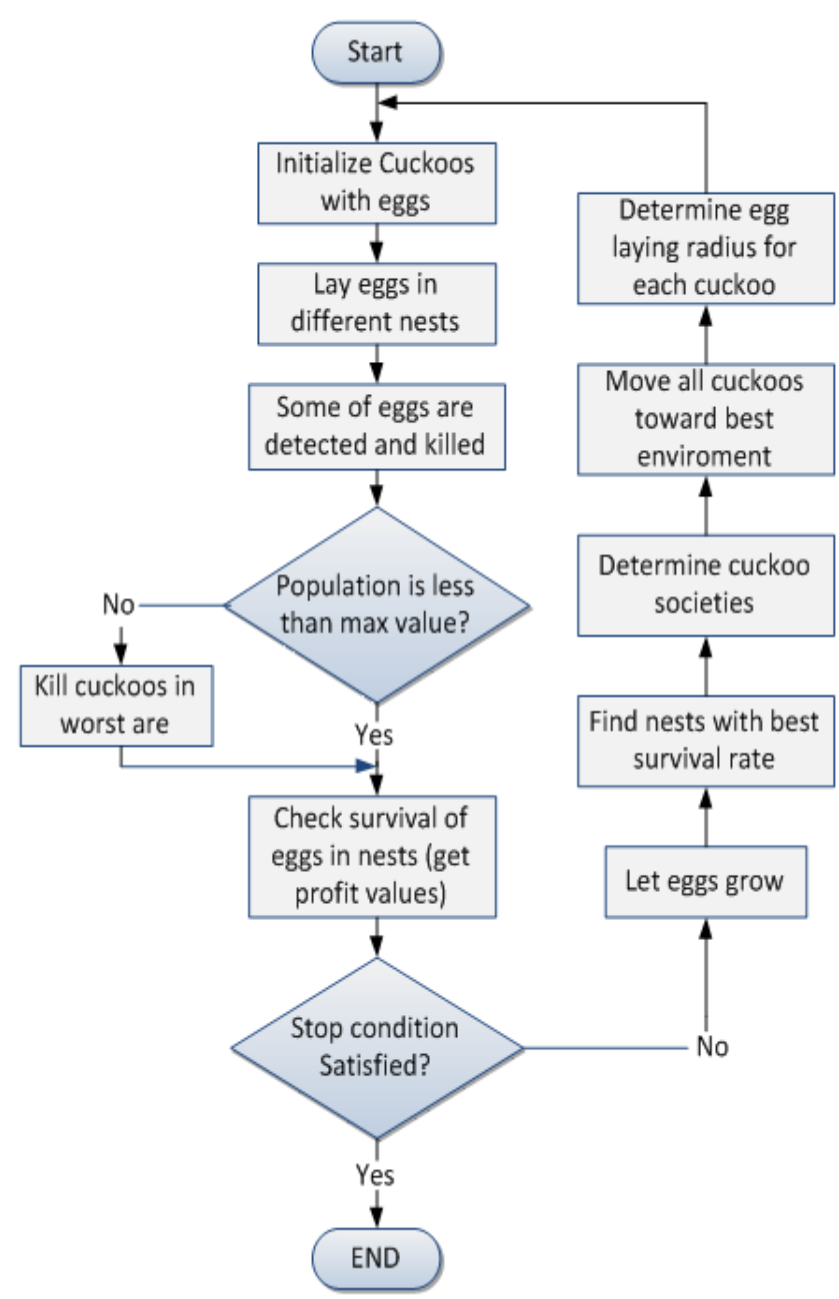

Fig.1. Flowchart of COA method

\section{SIMULATION RESULTS}

In this section the case study for implementing the framework and the simulation results are presented. The test casew is a 13-bus test system as it is shown in Fig.2. The system information was obtained from the $63 / 20 \mathrm{kV}$ KhodaBande-Loo substation of the Tehran distributed network. The bus and line information of the test system is given in the appendix. Bus information consists of active and reactive power connected to each bus. The total of active and reactive powers connected to busses are $10536 \mathrm{KW}$ and $5992 \mathrm{KVAR}$, respectively. Line information includes line resistance and reactance. According to the size of the network and power loss in it, renewable resources are locate as the DG source.

As mentioned above, the aim of this paper is to find the optimal location and capacity of DG according to the factor weight of each parameter using the COA. The main purpose 
is identify if a specific parameter is more important than other parameters for DG capacity and location in different conditions and places. Five scenarios are considered for this case:

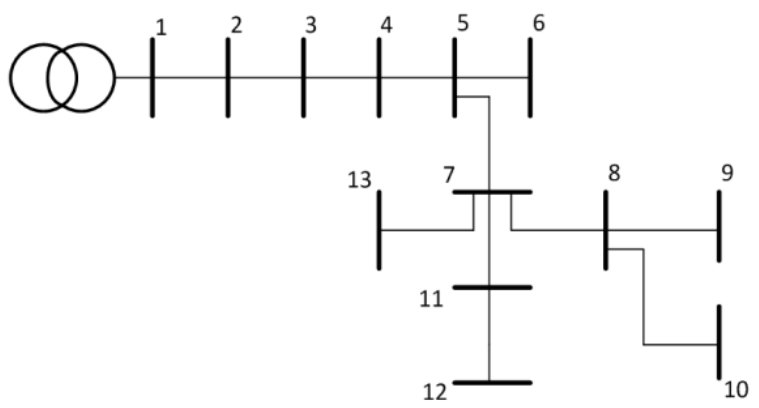

Fig.2. The 13 bus system case study

Case I: The first scenario is the equal weight for all factors. The three parameters, namely, loss reduction, voltage profile, and DG fuel cost, are equally important, i.e.,

$$
K_{1}=K_{2}=K_{3}=\frac{1}{3}
$$

Case II: In the second scenario, it is assumed that the DG fuel cost factor has a higher weight than the losses and voltage profile, and weight of these factors are:

$$
K_{1}=K_{2}=0.2, K_{3}=0.6
$$

Case III: In the third scenario it is assumed that the distribution system is related to consumers with a high degree of importance, therefore the parameter of voltage profile has the most important, and subsequently the losses parameter is also more important and DG fuel costs have a lower priority.

$$
K_{1}=0.3, K_{2}=0.6, K_{3}=0.1
$$

Case IV: In the fourth scenario, as in the second scenario, the DG fuel prices factor has a higher weight factor and is equal to the sum of the other two.

$$
K_{1}=0.25, K_{2}=0.25, K_{3}=0.5
$$

Case V: Also, in the fifth scenario

$$
K_{1}=0.05, K_{2}=0.45, K_{3}=0.5
$$

In this paper, all the simulations are programmed in MATLAB. The results of the simulations are shown in Tables 1 through 5 for every change in parameters. As it is evident from the results, when the parameter weight of DG cost increases, The DG size decreases. For example, as it can be seen from Table 2, by increasing the weight parameter (K3) to 0.6 , the DG size decreases to zero. In Tables 2, 4, and 5 DG parameter weights are more important than other parameters. For this purpose, the DG generation is less than other tables. In Table 4 , the coefficients $\mathrm{K}_{1}$ and $\mathrm{K}_{2}$ are equal to 0.25 but in Table 5 these coefficients are 0.05 and 0.45 , respectively. As it can be seen from these tables, because the loss parameter weight in Table 4 is more than Table 5 the amount of loss is greater. In addition, it can be seen in Fig. 3 that because of voltage improvement the parameter weight in Table 5 is more than Table 4, and the voltage profile has a better condition. Moreover, an increase in K3 causes the voltage profile to be placed in a worse situation.

Fig. 4 demonstrates the amount of loss reduction for the five scenarios. In addition, Fig. 5 demonstrates the penetration of DG sources for different scenarios. As it can be seen from these figures, loss reduction is increased when DG penetration raises in the system.

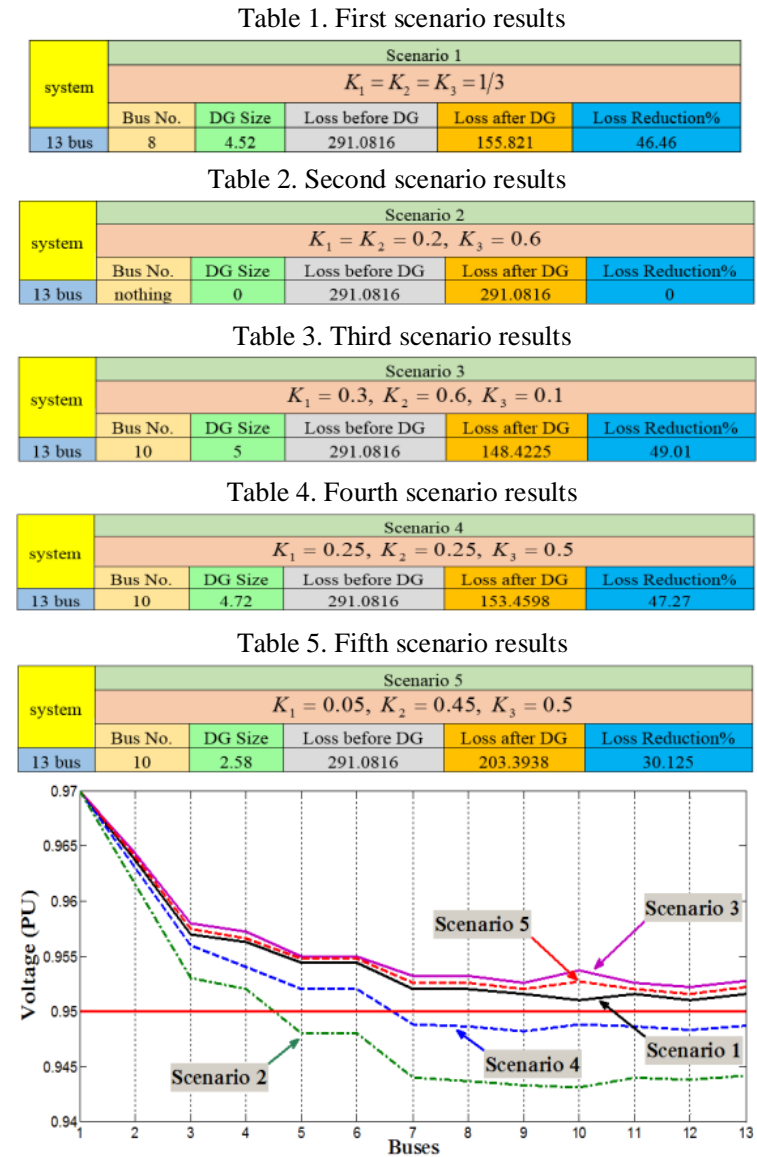

Fig. 3. Voltage profile in the network for different scenarios

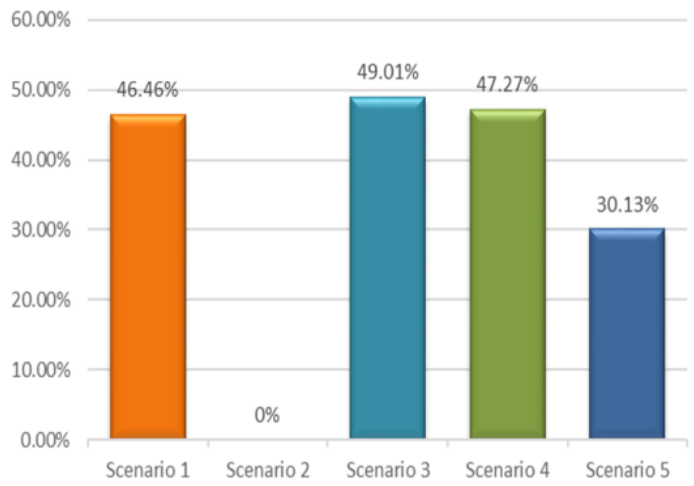

Fig. 4. Loss reduction in different scenarios 


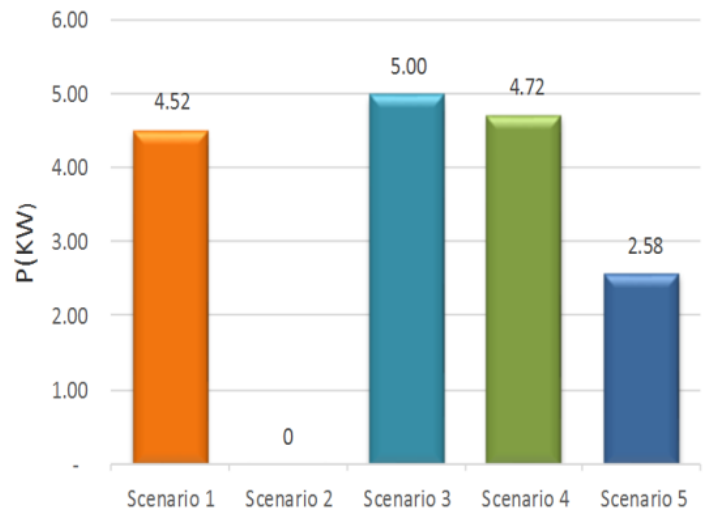

Fig. 5. DG penetration in different scenarios

\section{CONCLUSION}

This paper investigated the effects of varying the weight of parameters to determine the location and size of the DG. To achieve this goal, an evolutionary optimization algorithm called cuckoos optimization algorithm (COA) was deployed. It was shown that the proposed algorithm worked well according to various conditions. It was found that loss parameter weight and improve voltage profile weight influence each other. On the other hand, improvement in one of these parameters caused the relative improvement to the other. However, the third parameter weight, DG fuel cost, had a devastating influence on both the two other parameters and increasing this parameter caused all of the other parameters to have worse conditions.

\section{REFERENCES}

[1] A Aranizadeh, B Vahidi, A Rahiminejad, "Wind turbine power output smoothing in microgrid using ultra-capacitor with continuous wind speed forecasting and online supervisory control", Journal of Renewable and Sustainable Energy, Vol. 8, (3), 2016.

[2] K. Y. Lee, Xiaomin Bai, Young-Moon Park, Optimization method for reactive power planning by using a modified simple genetic algorithm, IEEE transactions on power system, November 1995, v. 10, pp. 1843-1850.

[3] S. Aznavi, P. Fajri and A. Asrari, "Smart Home Energy Management Considering Real-Time Energy Pricing of Plug-in Electric Vehicles," in 2018 IEEE Energy Conversion Congress and Exposition (ECCE), Portland, OR, USA, 2018, pp. 67-72.

[4] Niazazari, I., Vahidi, B., \&Abyaneh, H. A. (2013), Loss reduction of wind turbine with optimization of blade length using genetic algorithm. Science International, 25(4), pp. 807 -811.

[5] H. Hedayati, S. A. Nabaviniaki, A. Akbarimajd, A method for placement of DG units in distribution networks, IEEE transactions on power delivery,July 2008, v. 23, pp. 1620-1628.

[6] O. A. Gashteroodkhani, M. Majidi, M. Etezadi-Amoli, "A Fuzzybased Control Scheme for Recapturing Waste Energy in Water Pressure Reducing Valves" IEEE Power and Energy Society General Meeting (PESGM), pp. 1-5, Portland, OR, Aug 2018.

[7] I. Niazazari, H.A. Abyaneh, M. J. Farah, F. Safaei, andH. Nafisi,"Voltage profile and power factor improvement in PHEV charging station using a probabilistic model and flywheel," In Electrical Power Distribution Networks (EPDC), 2014 19th Conference on pp. 100-105. May. 2014

[8] X. Hugang, C. Haozhong, L. Haiyu, Optimal reactive power flow incorporating static voltage stability based on multi-objective adaptive immune algorithm, Energy conversion and management, May 2008, v. 49, pp. 1175-1181.

[9] D. Singh, D. Singh, K. S. Verma, Multiobjective optimization for DG planning with load models, IEEE transactions on power system, February 2009, v. 24, pp. 427-436.

[10] A. Rahiminejad, A. Aranizadeh, B. Vahidi, Simultaneous distributed generation and capacitor placement and sizing in radial distribution system considering reactive power market, Journal of renewable and sustainable energy, 2014, v. 6.
[11] A. Zeinalzadeh, Y. Mohammadi, M. H. Moradi, Optimal multi objective placement and sizing of multiple DGs and shunt capacitor banks simultaneously considering load uncertainty via MOPSO approach, Electrical power and energy systems, May 2015, v. 67, pp. 336-349.

[12] F. Safdarian and M. E. Nazari, "Optimal Sizing of a Solar-Thermal Collector for Residential Applications Using Gravitational Search Algorithm," Int. J. Mech. Eng. Autom, vol. 2, no. 11, pp. 497-504, 2015.

[13] K. Muthukumar, S. Jayalatitha, Optimal placement and sizing of distributed generators and shunt capacitors for power loss minimization in radial distribution networks using hybrid heuristic search optimization technique, Electrical power and energy systems, June 2016, v. 78, pp. 299-319.

[14] A. R. Phadke, M. Fozdar, K. R. Niazi, A new multi-objective fuzzyGA formulation for optimal placement and sizing of shunt FACTS controller, Electrical power and energy system, 2012, v. 40, pp. 4653

[15] L. J. Cai, I. Erlich, G. Stamtsis, Optimal choice and allocation of FACTS devices in deregulated electricity market using genetic algorithm, Power systems conference and exposition, October 2004, v. 1, pp. 201-207.

[16] M. Saravanan, S. Mary Raja Slochanal, P. Venkatesh, J. Prince Stephen Abraham, Application of particle swarm optimization technique for optimal location of FACTS devices considering cost of installation and system loadability, Electric power systems research, 2007, v. 77 , pp. $276-283$.

[17] O. A. Gashteroodkhani, M. Majidi, M. S. Fadali, M. Etezadi-Amoli, E. M. Amiri, "A Protection Scheme for Microgrids Using Time-Time matrix Z-score Vector", International Journal of Electrical Power and Energy Systems, vol. 110, pp. 400-410, 2019.

[18] R. S. Alabri, Ehab F. El-Saadany, Yasser M. Atwa, Optimal placement and sizing method to improve the voltage stability margine in adistribution system using distributed generation, IEEE transactions on power system, February 2013, v. 28, pp. 326-334.

[19] S. Aznavi, P. Fajri, M. Benidris, and B. Falahati, "Hierarchical droop controlled frequency optimization and energy management of a grid connected microgrid," in 2017 IEEE Conference on Technologies for Sustainability, Phoenix, AZ, USA, 2017, pp.1-7.

[20] V. Sarfi, I. Niazazari, and H. Livani, "Multiobjective fireworks optimization framework for economic emission dispatch in microgrids." North American Power Symposium (NAPS), 2016, pp. 1-6, Nov. 2016

[21] M. H. Moradi, M. Abedini, A combination of genetic algorithm and particle swarm optimization for optimal DG location and sizing in distribution systems, International journal of electrical power and energy systems, January 2012, v. 34, pp. 66-74.

[22] Juanuwattanakul, M. A. S. Masoum, Increasing distributed generation penetration in multiphase distribution networks considering grid losses, maximum loading factor and bus voltage limits, IET generation and distribution, December 2012,v. 6, pp. 1262-1271.

[23] B. Poornazaryan, P. Karimyan, G. B. Gharehpetian, M. Abedi, Optimal allocation and sizing of DG units considering voltage stability, losses and load variations, Electrical power and energy systems, July 2016, v. 79, pp. 42-59.

[24] M. Mirmozaffari, "Eco-Efficiency Evaluation in Two-Stage Network Structure: Case Study: Cement Companies". Iranian Journal of Optimization (IJO). Dec. 16, 2018.

[25] M. Mirmozaffari, "Developing an Expert System for Diagnosing Liver Diseases", EJERS, vol. 4, no. 3, pp. 1-5, Mar. 2019.

[26] M.Mirmozaffari and A. Alinezhad, " Ranking of Heart Hospital s Using cross-efficiency and two-stage DEA," $20177^{\text {th }}$ International Conference on Computer and Knowledge Engineering (ICCKE), Mashhad, 2017, pp. 217-222

[27] R. Rajabioun, Cuckoo Optimization Algorithm, In: Applied Soft Computing journal, Vol. 11, pp. 5508-5518, 2011 


\section{APPENDIX}

Table A1. Line information

\begin{tabular}{|c|c|c|c|}
\hline From & To & R (ohm) & X (ohm) \\
\hline 1 & 2 & 0.176 & 0.138 \\
\hline 2 & 3 & 0.176 & 0.138 \\
\hline 3 & 4 & 0.045 & 0.035 \\
\hline 4 & 5 & 0.089 & 0.069 \\
\hline 5 & 6 & 0.045 & 0.035 \\
\hline 5 & 7 & 0.116 & 0.091 \\
\hline 7 & 8 & 0.073 & 0.073 \\
\hline 8 & 9 & 0.074 & 0.058 \\
\hline 8 & 10 & 0.093 & 0.093 \\
\hline 7 & 11 & 0.063 & 0.05 \\
\hline 11 & 12 & 0.068 & 0.053 \\
\hline 7 & 13 & 0.062 & 0.053 \\
\hline
\end{tabular}

Table A2. Bus information

\begin{tabular}{|c|c|c|}
\hline Bus Number & P (kW) & Q (kVAR) \\
\hline 1 & 0 & 0 \\
\hline 2 & 890 & 468 \\
\hline 3 & 628 & 470 \\
\hline 4 & 1112 & 764 \\
\hline 5 & 1638 & 1378 \\
\hline 6 & 474 & 344 \\
\hline 7 & 1342 & 1078 \\
\hline 8 & 920 & 292 \\
\hline 9 & 766 & 498 \\
\hline 10 & 1662 & 480 \\
\hline 11 & 690 & 186 \\
\hline 12 & 1292 & 554 \\
\hline 13 & 1124 & 1480 \\
\hline
\end{tabular}

